\title{
On Jordan Ideals of Inverse Semirings with Involution
}

\author{
Liaqat Ali*, M. Aslam and Yaqoub Ahmed Khan \\ Department of Mathematics, Government College University, Lahore, Pakistan
}

\begin{abstract}
Article Type: Article
Article Citation: Liaqat Ali, M. Aslam, Yaqoub Ahmed Khan. On Jordan ideals of inverse semirings with involution. Indian Journal of Science and Technology. 2020; 13(04), 430-438. D0l: 10.17485/ijst/2020/ v013i04/149311
\end{abstract}

Received date: November 25, 2019 Accepted date: December 5, 2019

*Author for correspondence: Liaqat Ali Vrehmani_Ihr@yahoo. com 9 Department of Mathematics, Government College University, Lahore, Pakistan

\begin{abstract}
Objectives: The main objective of this article is to introduce *-Jordan ideals of a certain class of semirings called MA-semirings with involution and to investigate some conditions for which the above said ideals contained in the center. Methods and findings: We use the Jacobian identities and 2-torsion freeness of MA semirings. In this connection, we establish some important results of ring theory for the class of MA-semirings. Applications/ improvements: The commutative property is helpful to study the theory of semirings with ease therefore we find some conditions to impose commutativity in semirings, which are indeed novel idea in the field of semirings. Furthermore, these conditions are used in a most generalized way that these conditions bring the *-Jordan ideals to the center, therefore, it would be the corollary of result that semiring is commutative.

Keywords: Semirings, *-Semirings, MA-Semirings, *-Jordan Ideals, *-Prime Semirings, Involution Mathematics Subject Classifications (2010): 16Y60, 16W10
\end{abstract}

\section{Introduction and Preliminaries}

In literature, usually a self-inverse antiautomorphism for a given algebraic system is referred as involution [1-6]. It is worthy to be noted that the study of semirings with involution was also focussed by many authors in di_erent aspects. The notion of semirings was introduced by authors in Refs. [7-8] introduced the concept of inverse semirings in 1974. In Ref. [9], Javed et al. studied additive inverse semirings satisfying $y\left(x+x^{\prime}\right)=\left(x+x^{\prime}\right) y, \forall x, y \in S$ i.e $x+x \in Z(S)$, where the $Z(S)$ is the center of $S$ and $x$ is the pseudo inverse of $x$, and they named such semirings as MA-Semirings. The theory of commutators along with derivations and certain additive mappings in MA-Semirings were further investigated [10]. The main aim of this study is to generalize some fundamental results of ${ }^{*}$-prime rings proved in Refs. [11-12] and investigate the condition for which the ${ }^{*}$-Jordan ideal is contained in the center of an MA-semiring. 
Now we include some necessary preliminaries for the sake of completeness. By a semiring $S$, we mean a semiring with absorbing zero ' 0 ' in which addition is commutative. A semiring $S$ is said to be additive inverse semiring if for each $x \in S$ there is a unique $x^{\prime} \in S$ such that $x+x^{\prime}+x=x$ and $x^{\prime}+x+x^{\prime}=x^{\prime}$. where $x^{\prime}$ is called the pseudo inverse of $x$. An additive inverse semiring $S$ is said to be an MA-Semiring if $x+x \in Z(S), \forall x \in S$. In fact every ring is MA-semiring while converse may not be true. The following is one of the examples of prime MA semiring which is not a ring. Such examples motivate us to generalize the results of ring theory in MA-semirings.

Example 1.1. The set $S=\{0,1,2,3, \ldots$.$\} with addition \oplus$ and multiplication $\odot$ respectively defined by $a \oplus b=\sup \{a, b\}$ and $a \odot b=\inf \{a, b\}$ is an MA-semiring. In fact, $S$ is a commutative prime MA-semiring.

Throughout the paper by a semiring $S$, we mean a proper MA-Semiring unless mentioned otherwise. $S$ is prime if $a S b=0$ implies that $a=0$ or $b=0$ and semiprime if

$a S a=0$ implies that $a=0 . S$ is 2 -torsion free if for $x \in S, 2 x=0$ implies $x=0$. An additive mapping *: $S \rightarrow S$ is an involution if $\forall x, y \in S,\left(x^{*}\right)^{*}$ and $(x y)^{*}=x^{*} y^{*}$. The notion of .*-primeness and *.-semiprimeness for inverse semiring is defined in Ref. [13]. An inverse semiring $S$ is *-prime if $a S b=0=a^{*} S b$ implies $a=0$ or $b=0$ and ${ }^{*}$-semiprime if $a S a=0=a^{*} S a$ implies $a=0$.

Example 1.2. Let $(S,+, \cdot)$ be an MA-semiring. Then the set $S$ with addition ' + ' and multiplication •defined as $a \bullet b=b . a$ forms an MA-semiring called the opposite MA-semiring of $S$. We usually denote it as $S_{O}$. Consider the set $R=S \times S_{O}$ with addition $\oplus$ and multiplication $\odot$ respectively defined as $(a, b) \oplus(c, d)=(a+c, b+d)$ and $(a, b) \odot(c, d)=(a . c ; b \bullet d)=(a c, d b)$. Then $(R, \oplus, \odot)$ forms an MA-semiring. Define $*: R \rightarrow R$ by $(x, y)^{*}=(y, x)$. Then *defines an involution on $R$. The MA-semiring $(R, \oplus, \odot)$ with involution *forms *-prime MA-semiring which is not prime. This example shows that a prime MA-semiring is a *-prime MA-semiring but converse is not true in general. An additive mapping $d: S \rightarrow S$ is a derivation if $d(x y)=d(x) y+x d(y)$. We define the commutator as $[x, y]=x y+y x, \forall x, y \in S$. By Jordan product, we mean $x \circ y=x y+y x, \forall x, y \in S$. An additive subsemigroup $G$ of $S$ is said to be a Jordan ideal if $x \circ r \in G, \forall x \in G, r \in R$ (see [14]). For all $\forall x, y, z \in S$ following identities hold which will be frequently used in the sequel:

$$
\begin{aligned}
& \left(I_{1}\right):[x y, z]=x[y, z]+[x, z] y, \quad\left(I_{2}\right):[x, x y]=x[x, y], \\
& \left(I_{3}\right):[x, y z]=[x, y] z+y[x, z], \quad\left(I_{4}\right):(x y)^{\prime}=x y=x y^{\prime}, \\
& \left(I_{5}\right):[x, y]^{\prime}=\left[x, y^{\prime}\right]=\left[x^{\prime}, y\right], \quad\left(I_{6}\right): x \circ(y+z)=x \circ y+x \circ z, \\
& \left(I_{7}\right):[x, y]+[y, x]=y\left(x+x^{\prime}\right)=x\left(y+y^{\prime}\right) .
\end{aligned}
$$

First, we introduce the notion of $*$-Jordan ideal for inverse semirings which is the generalization of the relevant concept of rings.

Definition 1.3. A Jordan ideal $G$ is *-Jordan ideal of $S$ if $G^{*}=G$.

Definition 1.4. An element $x \in S$ is hermitian if $x^{*}=x$. and skew hermitian if $x^{*}=x^{\prime}$. The sets of all hermitian and skew hermitian elements are denoted by $\mathrm{H}$ and $\mathrm{K}$, respectively and $S_{a *}(S)=H \cup K$. 
We can easily establish the following lemma.

Lemma 1.5. Let $S$ be a semiring with involution $*$ and $d: S \rightarrow S$ be a derivation. Then for any $x, y \in S$, we have the following

$$
(i) \cdot\left(x^{*}\right)^{\prime}=\left(x^{\prime}\right)^{*}(i i) \cdot d\left(x^{\prime}\right)=(d(x))^{\prime}(i i i) \cdot\left[x^{*}, y^{*}\right]=[y, x]^{*}
$$

\section{Main Results}

Lemma 2.1. Let $G$ be a nonzero Jordan ideal of a semiring $S$. Then $2[S, S] G \subseteq G$ and $2 G[S, S] \subseteq G$.

Proof. Let $r, s \in S$ and $j \in G$. Then by the definition of Jordan ideal we have $j \circ[r, s] \in G, j \circ r \in G,(j \circ r) \circ s \in G$ and $\quad(j \circ s) \circ r \in G$. As $\quad s^{\prime} \in S$, therefore $j \circ[r, s]+(j \circ r) \circ s^{\prime}+(j \circ s) \circ r \in G$. By using definitions, we have $j \circ\left(r s+s^{\prime} r\right)+(j r+r j) \circ s^{\prime}$ $+(j s+s j) \circ r \in G$. As $S$ is an MA-semiring, therefore $j s^{\prime} r+r s j+j s r+r s j+2 s^{\prime} r j \in G$. The use of definition of MA-semiring and simplification imply that $2\left(r s+s^{\prime} r\right) j \in G, \forall r, s \in S, j \in G$ and hence $2[r, s] j \in G, \forall r, s \in S, j \in G$. This proves that $2[S, S] G \subseteq G$.

Using the similar arguments, we can show that $2 G[S, S] \subseteq G$.

Lemma 2.2. Let be a nonzero *-Jordan ideal of a 2-torsion free $*$-prime semiring $S$. If $a, b \in S$ such that $a G b=a^{*} G b$, then either $a=0$ or $b=0$.

Proof. We have

$$
a G b=0=a^{*} G b \text { for some } a, b \in S
$$

Suppose $a \neq 0$. In view of lemma 2.1 , we have $2[r, s] j \in G, \forall r, s \in S, j \in G$. As $S$ is 2-torsion, from (1) we have for all $r, s \in S, j \in G$

$$
a[r, s] j b=0
$$

In (2) replacing $s$ by $s a$ we have $a[r, s a] j b=0$. Using above identities and (2) again, we get $a s[r, a] j b=0$. Using (1), we get $a s a^{\prime} r j b=0$, which implies $a s a r j b=0$ and hence

$$
a S a r j b=0, \forall r \in S, j \in G
$$

From $a^{*} G b=0$, in the similar fashion, we get

$$
a^{*} \operatorname{Sarjb}=0
$$

As $a \neq 0$, using *-Primeness of $S$, in view of (3) and (4), we obtain

$$
a r j b=0
$$

In (2), replacing $s$ by $s a^{*}$, we obtain $a\left[r, s a^{*}\right] j b=0, \forall r, s \in S, j \in G$. Using (1) and simplifying we get $a s a^{*} r^{\prime} j b=0$ and hence

$$
a S a^{*} r^{\prime} j b=0
$$

Again from (1), we can write $a^{*}\left[r, s a^{*}\right] j b=0, \forall r, s \in S, j \in G$, which further gives

$$
a^{*} S a^{*} r j b=0
$$


Using *-primeness of $S$, in view of (6) and (7), we get

$$
a^{*} S j b=0
$$

Using *-primeness of $S$ in (5) and (8), we get

$$
G b=0
$$

As $G$ is a Jordan ideal, in view of (9), we have $(j \circ r) b=0$. Consequently, $j r b=0$ and hence

$$
j S b=0
$$

As $G$ is *-invariant, therefore

$$
j^{*} S b=0
$$

In view of *-primeness of $S$ as $G \neq 0$, using (10) and (11), we get $b=0$, which completes the proof.

Following result is a generalization of Lemma 3 of [5].

Theorem 2.3. Let $G$ be a nonzero *-Jordan ideal of a 2-torsion free *-prime semiring $S$. If $[G, G]=0$, then $[S, G]=0$ and so $G \subseteq Z(S)$.

Proof. For all $x, y \in G$, we have

$$
[x, y]=0
$$

In view of lemma 2.1, replacing $x$ by $2 x[r, s], x \in G, r, s \in S$ in (12) and since $S$ is 2 -torsion free, we obtain $[x[r, s], y]=0$. Using the above identities and (12) again, we obtain $x[[r, s], y]=0$ and hence

$$
G[[r, s], y]=0
$$

By using the same arguments as of theorem 2.2 equation (9), we get

$$
[[r, s], y]=0
$$

Replacing $s$ by $s r, r, s \in S$ in (14), we get $[[r, s r], y]=0$. By using the above identities and again using (14), we get

$$
[r, s][r, y]=0
$$

Replacing $s$ by $x s$ in (15) and using (15) again, we get $[r, x] s[r, y]=0$ and hence for all $x, y \in G, r, s \in S$

$$
[r, x] S[r, y]=0
$$

Since $G=G^{*}$, therefore, replacing $y$ by $y^{*}$ in (16), we get

$$
[r, x] S[r, y *]=0
$$


For any $r \in S a_{*}(S)(17)$ becomes

$$
[r, x] S[r, y]^{*}=0
$$

By the *-primeness of $S$, using (16) and (18), we obtain

$$
[r, x]=0
$$

As $\left(r *^{\prime}+r\right) \in S a_{*}(S), \forall r \in S$, simplifying through the above identities and using (19), we get

$$
[r, x]=\left[r^{*}, x\right], \forall x \in G, r \in S
$$

Replacing $r$ by $r^{*}$ in (17), we get $\left[r^{*}, x\right] S\left[r^{*}, y^{*}\right]=0, \forall x, y \in G, r, s \in S$. By using (20) and Lemma 1.5 (iii), we get

$$
[r, x] S[r, y]^{*}=0
$$

In view of *-Primeness of $S$, using (16) and (21), we get $[r, x]=0$, therefore, $[G, S]=0$ and hence $G \subseteq Z(S)$.

Following result is a generalization of Lemma 4 of [5].

Theorem 2.4. Let $G$ be a nonzero *-Jordan ideal of a 2 -torsion free *-prime semiring $S$ and $d$ a derivation of $S$ satisfying $d(G)=0$. Then $d=0$ or $[G, S]=0$.

Proof. By the hypothesis for any $j \in G$ we have

$$
d(j)=0
$$

As $G$ is *-Jordan ideal for any $j \in G, r \in S$ we have $j \circ r \in G$ and therefore $d(j \circ r)=0$. Using (22), we obtain

$$
d(r) j+j d(r)=0
$$

In (23) replacing $r$ by $r s, s \in S$ after simplification, we get $j(d(r) s+r d(s))+(d(r) s+r d(s)) j=0$.

From (23), using $j d(r)=d(r) j^{\prime}$, we get $d(r) j^{\prime} s+d(r) s j+j r d(s)+r j^{\prime} d(s)=0$ and therefore

$$
d(r)[s, j]+[j, r] d(s)=0
$$

Replacing $s$ by $g \in G$ in (24) and using (22), we get

$$
d(r)[g, j]=0
$$

In (25) replacing $r$ by $r t, t \in S$ and again using (25), we get $d(r) t[g, j]=0$ and so

$$
d(r) S[g, j]=0
$$


Since $G=G^{*}$, from (26) we have

$$
d(r) S[g, j]^{*}=0
$$

By the *-primeness of $S$, using (26) and (27), we get that either $d=0$ or $[G, G]=0$. As $d$ is nonzero, by Theorem 2.3, $[G, S]=0$, we get $G \subseteq Z(S)$.

Following result is a generalization of Theorem 1 of [5].

Theorem 2.5. Let $G$ be a non-zero *-Jordan ideal and subsemiring of a 2-torsion free *-prime semiring $S$. If $d$ is a nonzero derivation that commutes with $*$ and satisfies either $d(j k)+(d(j))^{\prime} d(k)=0$ or $d(j k)+(d(k))^{\prime} d(j)=0$, then $G \subseteq Z(S)$.

Proof. By the hypothesis $d(j k)+(d(j))^{\prime} d(k)=0 \forall j, k \in G$ i.e

$$
d(j) k+j d(k)+(d(j))^{\prime} d(k)=0
$$

In (28) replacing $k$ by $k l$, we get $d(j) k l+j d(k l)+(d(j))^{\prime} d(k l)=0$. After simplification and using (28) again, we get $\left(j+(d(j))^{\prime}\right) k d(l)=0$ i.e.

$$
\left(j+(d(j))^{\prime}\right) G d(l)=0
$$

As $G=G^{*}$ and $d$ commutes with *, therefore replacing $l$ by $l^{*}$ in (29), we have

$$
\left(j+(d(j))^{\prime}\right) G(d(l))^{*}=0
$$

In view of theorem 2.2, using (29) and (30), we have either $\left(j+(d(j))^{\prime}\right)=0$ or $d(l)=0$.

Case-I: If $j+(d(j))^{\prime}=0$. As $\mathrm{G}$ is subsemiring, therefore replacing $j$ by $j k$, we get $j k+\left(d(j k)^{\prime}\right)=0$. By using $j+(d(j))^{\prime}=0$, we get $j=d(j)$, therefore the last equation becomes

$$
j k
$$

In (31) replacing $k$ by $r \circ k$ where $r \in S$, we get $j(r \circ k)=0$ and using (31) again, we get jrk $=0$, which implies that

$$
j S k=0
$$

Since $G=G^{*}$, therefore from (32) we have

$$
j S k^{*}=0
$$

By the ${ }^{\star}$-primeness of $S$, using (32) and (33), we get $G=0$, a contradiction.

Case-II: If $d(l)=0$ i.e $d(G)=0$. By Theorem 2.4, we have $G \subseteq Z(S)$ since $d$ is nonzero. Secondly, assume that $d(j k)+(d(k))^{\prime} d(j)=0$ and therefore

$$
d(j) k+j d(k)+(d(k))^{\prime} d(j)=0
$$


In (34) replacing $j$ by $j k$, we get $d(j k) k+j k d(k)+(d(k))^{\prime} d(j k)=0$. Simplifying and using (34) again, we get

$$
j k d(k)+(d(k))^{\prime} j d(k)=0
$$

Replacing $j$ by $l j$ in (35), we get

$$
l j k d(k)+(d(k))^{\prime} l j d(k)=0
$$

From (35), we have $j k d(k)=d(k) j d(k)$, therefore (36) becomes $\left(l d(k)+(d(k))^{\prime} l\right) j d(k)=0$ or $[d(k), l] j d(k)=0$, which implies that

$$
[d(k), l] G d(k)=0
$$

Since $d$ and $*$ commute, therefore for any $k \in G \cap S a_{*}(S)$, we have

$$
[d(k), l] G(d(k))^{*}=0
$$

By Lemma 2.2, using (37) and (38), we get either $[d(k), l]=0$ or $d(k)=0$. For any $k \in G$, one can easily observe that $k^{*^{\prime}}+k \in G \cap S_{a_{*}}(S)$, therefore, we have either $d\left(k^{*^{\prime}}+k\right)=0$ or $\left[d\left(k^{*^{\prime}}+k\right), l\right]=0$. If $d\left(k^{*^{\prime}}+k\right)=0$, then $d\left(k^{*^{\prime}}\right)+d(k)=0$ and consequently $(d(\vec{k}))=d(k)$ and therefore $d(k) \in S_{a_{*}}(S)$. Therefore, (37) becomes

$$
[d(k), l] G(d(k))^{*}=0
$$

By lemma 2.2, using (37) and (39), we get either $d(k)=0$ or $[d(k), G]=0, \forall k \in G$ .Secondly, if $\left[d\left(k^{*^{\prime}}+k\right), l\right]=0$, then

$$
\left[d\left(k^{*}\right), l\right]=[d(k), l]
$$

In (37) replacing $k$ by $k^{*}$, we get $\left.\left[d\left(k^{*}\right), l\right)\right] G d\left(k^{*}\right)=0$. Using (40) in the last equation, we get

$$
[d(k), l] G(d(k))^{*}=0
$$

In view of lemma 2.1, using (37) and (41), we get $d(k)=0$ or $[d(k), l]=0$ and therefore $d(k)=0$ or $[d(k), G]=0$. Consider the sets $G_{1}=\{k \in G: d(k)=0\}$ and $G_{2}=\{k \in G:[d(k), G]=0\}$. Clearly $G=G_{1} \cup G_{2}$. We claim that either $G=G_{1}$ or $G=G_{2}$ and for this we can show that either $G_{2} \subseteq G_{1}$ or $G_{1} \subseteq G_{2}$. Suppose that $j \in G_{1} \backslash G_{2}$ and $k \in G_{2} \backslash G_{1}$. We have $j+k \in G_{1}+G_{2} \subseteq G_{1} \cup G_{2}=G$. Therefore either $j+k \in G_{1}$ or $j+k \in G_{2}$. If $j+k \in G_{1}$, then $d(j)+d(k)=0$ and therefore $d(k)=0$ since $d(j)=0$, a contradiction. If $j+k \in G_{2}$, then $[d(j+k), l]=0, \forall l \in G$, therefore $[d(j), l]+[d(k), l]=0, \forall l \in G$. But since $k \in G_{2}$, therefore $[d(j), l]=0, \forall l \in G$ which implies $j \in G_{2}$, a contradiction. So, we 
conclude that $G_{2} \subseteq G_{1}$ or $G_{1} \subseteq G_{2}$. This means $G=G_{1}$ or $G=G_{2}$. If $G=G_{1}$, then $d(G)=0$ and therefore by Theorem 2.4 we have $\mathrm{G} \subseteq \mathrm{Z}(\mathrm{S})$. If $G=G_{2}$, then

$$
[d(j), k]=0
$$

In (42) replacing $j$ by $j k$, we get $[d(j k), k] \quad 0$ and using (42) again, we get

$$
[j, k] d(k)=0
$$

In (43) replacing $j$ by $j l$ and using (43) again, we get $[l, k] j d(k) \quad 0$ and therefore

$$
[l, k] G d(k)=0
$$

Using similar arguments as above us can write

$$
[l, k] G(d(k))^{*}=0
$$

Employing Lemma 2.2, (44) and (45) yield either $d(k)=0$ or $[k, G]=0, \forall k \in G$. Let $U_{1}=\{k \in G: d(k)=0\}$ and $U_{2}=\{k \in G:[d(k), G]=0\}$. Following the same process as performed earlier in this theorem, we get either $G=U_{1}$ or $G=U_{2}$. Hence we have either $d(G)=0$ or $[G, G]=0$. If $d(G)=0$, then by Theorem 2.4 we obtain $d=0$ or $G \subseteq Z(S)$. If $[G, G]=0$, then by Theorem 2.3 we have $G \subseteq Z(S)$. This completes the theorem.

\section{Conclusion}

This article introduces the notion of *-Jordan ideals of MA-semirings with involution* and presents some fundamental results in this regard. We specially focuses on deferent conditions satisfied by derivations on the above said ideals of a ${ }^{*}$-prime MA-semirings and extend some results of ${ }^{*}$-prime rings for the general class of ${ }^{*}$-prime MA-semirings. The concepts presented in this article have a lot of potential for nourishing. Therefore, this article is very useful as it invites the researcher's to explore more for other types of ideals and semirings.

\section{References}

1. Beidar KI, Martindale WS. On functional identities in prime rings with involution. Journal of Algebra. 1998; 203(2), 491-532. https://doi.org/10.1006/jabr.1997.7285.

2. Fajtlowicz S. Equationally completes semi groups with involution. Algebra Universalis. 1972; 1(1), 355-358. https://link.springer.com/article/10.1007/BF02944993.

3. Herstein IN. Rings with involution. University of Chicago Press: Chicago, Illinois. 1976. http:// www.sciepub.com/reference/73008.

4. Lanski C. Commutation with skew elements in rings with involution. Pacific Journal of Mathematics. 1979; 83(2), 393-399. https://projecteuclid.org/euclid.pjm/1102784518. 
5. Lee TK. On derivations of prime rings with involution. Chinese Journal of Mathematics. 1992; 20(2), 191-203. https://www.jstor.org/stable/43836467?seq=1.

6. Segal IE. Irreducible representations of operator algebras. Bulletin of the American Mathematical Society. 1947; 53(2), 73-88. https://projecteuclid.org/euclid.bams/1183510397.

7. Vandiver HS. Note on a simple type of algebra in which the cancellation law of addition does not hold. Bulletin of the American Mathematical Society. 1934; 40(12), 914-920. https:// projecteuclid.org/euclid.bams/1183497886.

8. Karvellas PH. Inversive semi rings. Journal of the Australian Mathematical Society. 1974; 18(3), 277-288. https://doi.org/10.1017/S1446788700022850.

9. Javed MA, Aslam M, Hussain M. On condition (A2) of Bandlet and Petrich for inverse semiqrings. International Mathematical Forum. 2012; 7(59), 2903-2914. https://www.researchgate.net/ publication/265986096_On_condition_A_2_of_Bandlet_Bandelt_and_Petrich_for_inverse_ semirings.

10. Sara S, Aslam M. On Jordan and Lie ideals in semirings. Hacettepe Journal of Mathematics and Statistics. 2019; 48(4), 996-1000. DOI: 10.1515/math-2017-0088.

11. Oukhtite L. On Jordan ideals and derivations in rings with involution, comment. Commentationes Mathematicae Universitatis Carolinae. 2010; 53(3), 389-395. https://eudml.org/doc/38135.

12. Zaidi SMA, Ashraf M, Ali S. On Jordan ideals and left $(\Theta, \Theta)$-derivations in prime rings. International Journal of Mathematics and Mathematical Sciences 2004(2004), 37-40, 1957-1964.

13. Ali L, Aslam M, Khan YA. Commutatively of semirings with involution. Asian-European Journal of Mathematics. November 2019. https://doi.org/10.1142/S1793557120501533

14. Sandhu GS, Kumar D. A note on derivations and Jordan ideals of prime rings. AIMS Mathematics. 2017; 2(4), 580-585. DOI: 10.3934/Math.2017.4.580. 